\title{
Bilinguals implicitly name objects in both their languages: an ERP study
}

\author{
Katie Von Holzen ${ }^{1,2 *}$ and Nivedita Mani ${ }^{2}$ \\ 1 Laboratoire Psychologie de la Perception UMR 8158, Université Paris Descartes, Paris, France \\ 2 Psychology of Language Research Group, Georg-Elias-Müller Institute of Psychology, University of Göttingen, Göttingen, Germany
}

\section{Edited by:}

lan FitzPatrick, Heinrich Heine

Universität Düsseldorf, Germany

Reviewed by:

Judith F. Kroll, Pennsy/vania State

University, USA

Jasmin Sadat, Royal Holloway,

University of London, UK

*Correspondence:

Katie Von Holzen, Laboratoire

Psychologie de la Perception UMR

8242, Université Paris Descartes,

75006 Paris, France

e-mail: katie.m.vonholzen@

gmail.com
Upon being presented with a familiar name-known image, monolingual infants and adults implicitly generate the image's label (Meyer et al., 2007; Mani and Plunkett, 2010, 2011; Mani et al., 2012a). Although the cross-linguistic influences on overt bilingual production are well studied (for a summary see Colomé and Miozzo, 2010), evidence that bilinguals implicitly generate the label for familiar objects in both languages remains mixed. For example, bilinguals implicitly generate picture labels in both of their languages, but only when tested in L2 and not L1 (Wu and Thierry, 2011) or when immersed in their L2 (Spivey and Marian, 1999; Marian and Spivey, 2003a,b) but not when immersed in their L1 (Weber and Cutler, 2004). The current study tests whether bilinguals implicitly generate picture labels in both of their languages when tested in their $L 1$ with a cross-modal ERP priming paradigm. The results extend previous findings by showing that not just do bilinguals implicitly generate the labels for visually fixated images in both of their languages when immersed in their L1, but also that these implicitly generated labels in one language can prime recognition of subsequently presented auditory targets across languages (i.e., L2L1). The current study provides support for cascaded models of lexical access during speech production, as well as a new priming paradigm for the study of bilingual language processing.

Keywords: bilingualism, implicit naming, phonological priming, lexical access, ERP

\section{INTRODUCTION}

Research on speech production has awarded considerable attention to the stages involved in a speaker's selection of an appropriate lexical item(s) to communicate her message. Among other issues, this work has examined how a speaker selects one word among other appropriate partially activated words for production, whether these other activated words interact with the speakers' choice and production of the chosen word, and the extent to which the phonological and semantic features of these other activated words are retrieved during speech production. Most models of speech production agree that the search for the appropriate lexical item in production also lends activation to items semantically related to the chosen word, either through activation of semantic features shared by the words or through activation of the corresponding lexical nodes of the semantically related words (Dell, 1986; Levelt, 1989; Roelofs, 1992; Caramazza, 1997). Models of speech production disagree, however, with regard to the extent to which the phonological features associated with these competing lexical nodes are retrieved in speaking. Discrete models of speech production suggest that while semantically related lexical nodes are simultaneously activated, phonological activation is restricted to the selected lexical node alone (Levelt, 1989; Levelt et al., 1999). In contrast, cascaded models of lexical access assume that the phonological properties of semantically related lexical nodes are all simultaneously activated (Dell, 1986; Caramazza, 1997; Dell et al., 1997).

Particularly useful for resolving the discrepancies between cascaded and discrete models is the study of bilingual speech production. Between their two languages, bilinguals have many translation equivalents with varying levels of phonological overlap. One class of words, cognates, contain similar orthographicphonological forms across languages. If, as argued by discrete models of speech production, only the phonological information for the corresponding node is activated, bilingual speech production should be similar for both cognate and non-cognate words. Studies investigating bilingual cognate and non-cognate picture naming, however, demonstrate a difference in naming latency between cognates and non-cognates (Costa et al., 2000; Hoshino and Kroll, 2008; Colomé and Miozzo, 2010; Strijkers et al., 2010; Poarch and van Hell, 2012). These results have overwhelmingly demonstrated that bilinguals activate phonological information from the non-target language, providing support for cascaded models of lexical access by showing that both selected and nonselected lexical nodes activate their corresponding phonological codes. Due to their special status across languages, however, the presence of cognate words may induce a bilingual processing mode (Wu and Thierry, 2010b). Stronger support for cascaded models of lexical access is therefore provided by studies not examining cognate word stimuli, yet still show that phonological information from both languages is activated during production in one language (Hermans et al., 1998; Colomé, 2001; Kaushanskaya and Marian, 2007; Hoshino and Thierry, 2011; Wu and Thierry, 2011). For example, Spalek and colleagues (Spalek et al., 2014) had German-English bilinguals produce adjective-noun pairs that either contained (e.g., green goat) or did not contain (e.g., green skirt) overt phonological onset overlap in English. Some trials, 
however, although they did not overlap in English, did contain phonological onset overlap once translated to German (e.g., blue flower, "blaue Blume"). Trials that overlapped overtly in English and covertly in German modulated the ERP (event-related potential) response in comparison to non-related trials, suggesting that German translation equivalents of the English words were simultaneously activated and influenced production, despite the entire experiment being conducted in English.

In the current study, we examine a special instance of the retrieval of phonological and semantic information in the selection of lexical nodes for production. Unlike the body of research described previously, which focused on overt production, we turn to covert production, or implicit label generation. This allows for the study of the information bilinguals use to name, or activate upon visual fixation of, objects, before information is ultimately chosen for production. Specifically, we examine whether bilingual speakers implicitly produce the labels of visually fixated images and whether they do so in both their languages.

Upon viewing an image, studies suggest that the label for this image is implicitly generated, and that this implicitly generated label can prime recognition of a subsequently presented, related target. In Meyer et al.'s study (Meyer et al., 2007; see also Jescheniak et al., 2002; Meyer and Damian, 2007), for example, adults were presented with an unlabeled prime image (i.e., boy) followed by a visual display of four images, one of which was a homophone of the unlabeled picture prime (i.e., buoy). There was no semantic overlap between the homophone target and the prime image. Indeed, the only overlap between the two images lies in the labels for the two images-any preference for looking toward the homophone image could, therefore only be explained as a result of participants' implicitly generating the label for both images and this implicitly generated label subsequently priming recognition of the related target image. Indeed, consistent with this explanation, participants were more likely to fixate the phonologically related target image compared to phonologically unrelated distractor images. This finding has been taken to show that participants implicitly generate the labels for visually fixated images, i.e., that they retrieve the phonological properties associated with the labels for visually fixated images. Such implicit label generation has also been found in infants using phonologically related prime-target pictures (Mani and Plunkett, 2010, 2011; Mani et al., 2012a), suggesting that auditory and visual information are integrated at as young as 18-months-of-age.

Implicit label generation has also been explored using the ERP method in a cross-modal priming paradigm. Desroches et al. (2009) presented participants with picture prime-spoken target word pairs that were either identical, onset-overlapping, rhymes, or unrelated, while simultaneously measuring participants' ERP responses to the spoken target words (see Mani et al., 2012b for similar studies with infants). ERPs (event-related potentials) are averaged waveforms of electrical brain activity (EEG) time-locked to the presentation of stimuli and can provide a measure of speech processing with a high temporal resolution of brain activity. One ERP component investigated by Desroches et al. (2009), the N400 (Kutas and Hillyard, 1984), described as a negative inflection peaking at approximately $400 \mathrm{~ms}$ after stimulus onset, indexes the integration of a stimulus into the context set by a preceding stimulus: the larger the N400 amplitude, the more difficult the integration process between stimuli. Although Desroches et al., reported some variation in component latency, N400 amplitude was reduced for both onset overlapping and rhyming prime-target pairs. Using a cross-modal priming procedure, the authors argued, ensures that any priming effects were the result of top-down processes resulting from connections at the phonological and lexical levels instead of bottom-up influence due to acoustic overlap between prime and target. This conclusion also assumes that participants implicitly generated the label for the picture primes, which ultimately primed recognition of the spoken target word.

Unlike monolingual speakers, however, bilingual speakers have at least two labels for every object, one in one language (e. g. dog, English) and one in the other (e. g. Hund, German). When viewing objects, therefore, bilinguals may implicitly generate the label in one or both of their languages. In terms of overt speech production in bilinguals, cross-language effects have been found when participants are tested in both their L1 and L2 and immersed in their L1 (Costa et al., 2000; Colomé, 2001; Hoshino and Kroll, 2008; Colomé and Miozzo, 2010; Strijkers et al., 2010; Poarch and van Hell, 2012) or their L2 (Hermans et al., 1998; Costa et al., 2000, 2009; Kaushanskaya and Marian, 2007; Hoshino and Thierry, 2011; Spalek et al., 2014). Explicit naming or overt production may tap into different processes compared to implicit naming or covert production, particularly to do with the later stages of the speech monitoring process involved in overt production and due to delays introduced by the actual production of muscle movements (see Wu and Thierry, 2011 for similar suggestions) which may allow the required time for effects of L2 access to appear. It is, therefore, important to distinguish between findings of studies examining explicit and implicit naming. With regard to covert production, however, some evidence suggests that bilinguals may implicitly generate the labels for objects in both their languages, but that this depends on the language of testing, as well as whether they are immersed in their L1 or L2 (see Wu and Thierry, 2010b for a discussion of context in bilingual experiments): Previous studies have demonstrated that bilinguals implicitly label objects in both languages when they are immersed in their L2 and tested in L2, but results differ when participants are tested in their L1 (Spivey and Marian, 1999; Marian and Spivey, 2003a,b; Wu and Thierry, 2011). In the current study, using the cross-modal priming paradigm of Desroches et al. (2009), we examine whether bilinguals implicitly generate the label for objects in both of their languages when they are tested in their L1 and immersed in an L1 environment, a context that has previously failed to yield this effect (Weber and Cutler, 2004).

For bilinguals immersed in an L2 environment, successful L2 performance may come at the cost of L1 fluency. Linck et al. (2009) compared English learners of Spanish who were either immersed in a Spanish, L2 environment, or remained in their native, L1 English environment. When tested on both comprehension and production, an interesting asymmetry appeared between the two groups of participants: although the L2 performance was better for learners immersed in an L2 environment than their L1 environment counterparts, these participants showed decreased L1 access. This pattern of results led the authors to suggest that when immersed in their L2, the learners inhibited 
activation of their L1. Within a group of participants tested before and after L2 immersion, however, Baus et al. (2013) found similar results, although only for low frequency, non-cognate words, suggesting that the decrease in L1 access during L2 immersion is the result of decreased L1 usage and not L1 inhibition. Although the purpose of the current paper is not to resolve which mechanisms are at work during L2 or L1 immersion, these studies highlight the effects that immersion can have on L1 and L2 access and performance.

To our knowledge, only one study has specifically investigated the question of whether bilinguals implicitly generate the label in one or both of their languages (although others have indirectly addressed it, see below). Wu and Thierry (2011) presented Chinese-English participants with pairs of pictures and asked them to judge whether the labels of the two pictures rhymed in L2 English (Experiment 1) or shared a character in L1 Chinese (Experiment 2).The stimuli were manipulated such that some of the picture pairs overlapped in one language (e. g. rhymed in L2 English), while others overlapped in the other language (e. g. character overlap in L1 Chinese). EEG data was recorded throughout the experiment to examine the neurocognitive indices of cross-language lexical access. Consistent with the standard N400 priming effect (Kutas and Hillyard, 1984), when asked to evaluate L1 Chinese overlap, participants found picture pairs whose labels overlapped in Chinese easier to process relative to unrelated picture pairs. Similarly, when asked to evaluate L2 English overlap, participants found picture pairs whose labels rhymed in English easier to process relative to unrelated pictures pairs whose labels did not rhyme. Critically, when asked to evaluate L2 English overlap, picture pairs whose labels overlapped in L1 Chinese were also easier to process, suggesting that ChineseEnglish bilinguals activated both the L1 and L2 labels for the pictures. However, an effect of L2 English overlap was not found when participants were making rhyme judgments in L1 Chinese. Wu and Thierry attribute this asymmetric effect to the possibility that L2 word forms are not implicitly generated while making judgments in L1. In contrast, L1 word forms were activated during $\mathrm{L} 2$ processing and $\mathrm{Wu}$ and Thierry suggest that this is the result of bilinguals' inability to prevent interference from their L1 during L2 speech planning (Green, 1998).

Experiments using the visual world paradigm, however, suggest that both languages are activated even when bilingual participants are tested in their L1 and immersed in an L2 environment. In a series of experiments, Marian and Spivey (2003a,b) and Spivey and Marian (1999) presented Russian-English bilinguals with a visual display containing several objects. In one version of the experiment, participants were instructed in L1 Russian to move a target object (e. g. marka, "stamp"). Although they were tested in Russian, participants were more likely to look at a distractor object that had a phonologically related label in L2 English (e.g., flomaster, "marker") than a distractor object with an unrelated label (e.g., lineka, "ruler"). The results suggest that the word form of objects were also activated in L2 English, causing the English label for the phonologically related distractor object (i.e., flomaster, "marker") to compete for activation with the Russian label for the target object (i.e., marka, "stamp"). In another version of the experiment, where participants were instructed in L2
English, they were more likely to look at a target that was phonologically related in L1 Russian, than a distractor object with an unrelated label. In other words, bilinguals implicitly generated the label for the objects in both of their languages, regardless of the language of testing.

Nevertheless, participants in the Marian and Spivey experiments were immersed in their L2, where they heard their L2 every day in their surrounding environment. Using a similar paradigm to that of Marian and Spivey, Weber and Cutler (2004) extended the results of Marian and Spivey to participants tested in their L2 while immersed in their L1. Interestingly, Weber and Cutler did not find evidence of L2 activation when participants were tested in their L1 while immersed in an L1 environment. Weber and Cutler suggest that these results may reflect a difference in the background of their participants and the testing environment in comparison to the bilinguals tested by Spivey and Marian: The bilinguals tested by Spivey and Marian were immersed in their L2, English, perhaps increasing the likelihood that English would be co-activated. In contrast, the Dutch-English bilinguals tested by Weber and Cutler lived in the Netherlands and used their L1, Dutch, in their everyday life, making L2 English less relevant for activation when participants were tested in L1. It may, therefore, be more likely for L2 words to be activated during L1 processing when the participants are immersed in their L2.

It is of interest, however, that the Chinese-English bilinguals tested by Wu and Thierry (2011) do not show effects of L2 activation in L1 processing, despite being immersed in their L2 (similar to the language environments of Spivey and Marian, 1999; Marian and Spivey, 2003a,b). A potential explanation for this difference might come from nature of the task performed by participants. Spivey and Marian did not explicitly ask participants to judge the phonological overlap between target and distractor object labels in either of the languages of the participants, while $\mathrm{Wu}$ and Thierry focused participants' attention on phonological overlap for the picture pairs in one language, i.e., either their L1 or their L2. It is possible that this conscious focus on phonological overlap in the one language may reduce the influence of the "other" language, especially when the other language is the less dominant L2. The current study, therefore, does not focus participants' attention on phonological overlap in either of their languages. Instead, we asked participants to perform a non-linguistic task (a picture matching task), which drew their attention away from the relationship between the prime and target. We suggest that this provides a more accurate measure of whether bilinguals implicitly generate the labels of visually presented images in both their languages by not biasing their attention to linguistic relationships.

Using a cross-modal priming paradigm, participants were presented with visual picture primes (presented in silence) followed by auditory L1 targets. Although it differs from the picturepicture task of $\mathrm{Wu}$ and Thierry, it is similar to the visual world paradigm (Spivey and Marian, 1999; Marian and Spivey, 2003a,b; Weber and Cutler, 2004) where the target is a spoken word. This paradigm, shown to elicit an N400 component for both onset and rhyme related picture prime-target word pairs (Desroches et al., 2009), allows not only for the study of implicit label generation, but also removes the potential role of acoustic overlap between prime and target. This also allowed for an unbiased 
investigation of cross-linguistic priming on auditory word recognition (i.e., L2 picture prime label—L1 auditory target). Although studies that have investigated auditory word recognition in bilinguals are increasing (Sinai and Pratt, 2002; Ju and Luce, 2004; Weber and Paris, 2004; Blumenfeld and Marian, 2005, 2007; Cutler et al., 2006; Marian et al., 2008; Rueschemeyer et al., 2008; Canseco-Gonzalez et al., 2010; FitzPatrick and Indefrey, 2010; Altvater-Mackensen and Mani, 2011; Lagrou et al., 2011; see also Shook and Marian, 2012; Weber and Broersma, 2012; Von Holzen and Mani, 2012; FitzPatrick and Indefrey, 2014), there are relatively few studies that have specifically investigated whether cross-linguistic priming can influence auditory word recognition (Phillips et al., 2006; Pratt et al., 2013). These studies, however, used both languages within their experiment, possibly creating an artificial bilingual environment (Grosjean, 1997). In the current study, we address this problem by testing participants exclusively in their L1.

In the current study, implicit generation of both language labels was examined by manipulating the relationship between the L1 and L2 labels for the picture prime and the L1 auditory target words. Thus, we included four conditions in the experiment: (1) picture prime labels and L1 target words were identical in L1 German; (2) L1 German labels for the picture primes and L1 target words were phonologically related in German; (3) L2 English labels for the picture primes and L1 target words sounded similar $^{1}$; (4) L1 and L2 labels for the picture prime and L1 target words were phonologically, orthographically, or semantically unrelated (see Figure 1 for examples). Similar to Wu and Thierry (2011), in trials where picture prime labels and L1 target words were related within- or between-language, the relationship was offset-overlap, which has already been demonstrated to elicit a N400 priming effect in a similar study with monolingual participants (Desroches et al., 2009). In summary, the current study aims to answer two questions:

1. When viewing images, do bilinguals implicitly generate the labels for these images in one or both of their languages?

2. Can implicitly generated labels in L2 influence processing of auditory words in L1?

Our index of these effects is obtained from Event-related potential (ERP) recordings of participants' brain activity as they heard the auditory target labels, focusing on the N400 component. In the study of bilingualism, the N400 waveform has been used as a measure of priming between prime-target pairs that are related across languages semantically (Alvarez et al., 2003; Phillips et al., 2004; Martin et al., 2009; Palmer et al., 2010), orthographically (De Bruijn et al., 2001), phonologically (Altvater-Mackensen and Mani, 2011), as well as through their translations (Thierry and Wu, 2007; Wu and Thierry, 2010a, 2011; see Moreno et al., 2008 for a review of ERP use in bilingualism). We suggest that,

\footnotetext{
${ }^{1}$ As complete phonological overlap between words across languages rarely occurs (Dijkstra et al., 1999), reducing our stimuli set to prime-target pairs that overlap completely across languages would have severely reduced our word choice for prime-target pairs for the between-language related condition. As a result, we chose prime-target pairs that sounded similar to one another in English and German, respectively.
}

in contrast to the visual world paradigm, participants' ERPs may be a more sensitive index of the subtle differences involved in bilingual language processing (Mueller, 2005), given that participant eye gaze may be influenced by the number of objects in the visual display (Sorensen and Bailey, 2007) or simply not reflect competition effects (Dahan and Tanenhaus, 2004). In combination with the cross-modal priming paradigm, the use of ERPs may help clarify the differences in L2 activation during L1 processing when bilinguals are immersed in their L2 (Spivey and Marian, 1999; Marian and Spivey, 2003a,b) or their L1 (Weber and Cutler, 2004).

If participants were to implicitly generate the labels for the picture primes in their L1, this would be reflected by a significant reduction in N400 amplitude when the target word is either the same as the L1 label for the picture prime or phonologically related to the L1 label for the picture prime. Crucially, if bilinguals also implicitly generate the L2 labels for the picture primes, then N400 amplitude should also be reduced when the target word sounds similar to the L2 label for the picture prime. Furthermore, our manipulations allow for a a comparison of priming effects for prime-target pairs that are related within- (e.g., L1 to L1) and between-languages (e.g., L2 to L1). This relates to a question within the study of bilingualism which asks whether a bilinguals' two languages are organized into two separate, but connected lexicons or are integrated into one large lexicon and would present evidence of crosslanguage priming in an experiment conducted entirely in one language.

\section{MATERIALS AND METHODS PARTICIPANTS}

The current study tested a group of 16 German-English bilinguals (age $M=27.63$; age $S D=7.82$; age range $=20-48$ ). Participants were recruited as bilinguals from the local population in a middle-sized German city and told that the purpose of the experiment was to examine their visual perception and were afterwards debriefed on the full purpose of the experiment. After the experiment, participants filled out a language proficiency questionnaire (adapted from Rueschemeyer et al., 2008). In this questionnaire, participants indicated the age at which they began learning both German and English. All participants had been exposed to both German and English before age 10. Participants also indicated their proficiency in reading, writing, listening, speaking, and syntax in German and English. These proficiency scores were averaged to create a combined proficiency score for both German and English. Participants reported an average combined proficiency score that was similar in both German $(M=9.19 ; S D=1.02)$ and English $(M=9.05 ; S D=0.99 ; p>$ $0.05)$. In addition, participants also took part in a picture-naming task where we could assess the accuracy with which they labeled images in German and English. The results of these tests are reported in the Results section, showing that participants correctly and equally quickly labeled images in both German and English. All but three participants reported German to be their mother tongue and English their second language. Of these three, two participants reported German and English to be their mother tongue while one participant reported English to be her mother tongue, having learned German before she was 3 years of age. 


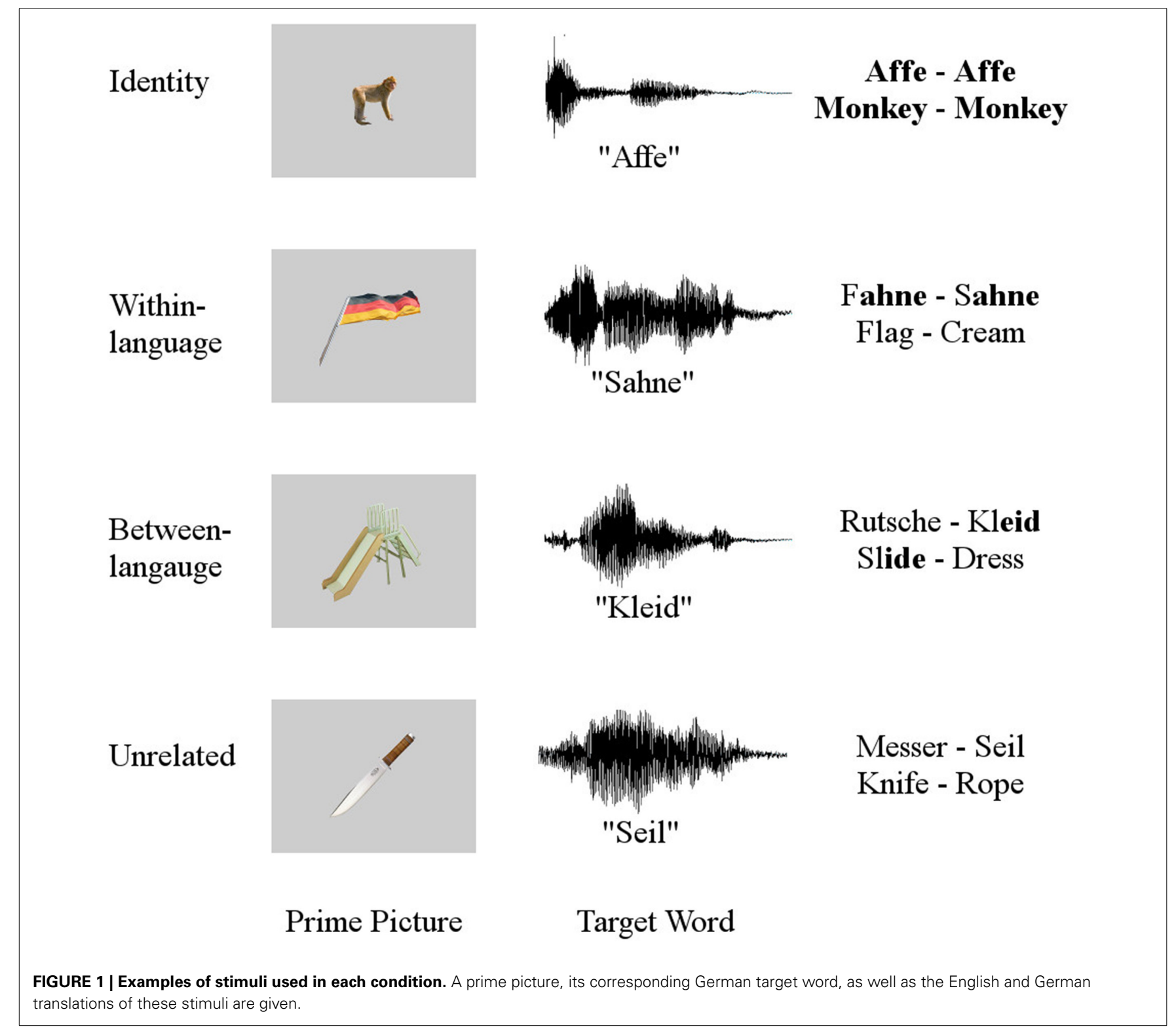

Therefore, we consider German to be the L1 of the participants and English, their L2, although these two languages are balanced. Participants were living in Germany at the point of testing, immersed in their L1. Before the experiment participants signed an informed consent form approved by the ethics committee of the University Göttingen and received 15 Euros afterwards for their participation.

\section{STIMULI}

The stimuli consisted of 120 primes and 120 targets, resulting in 120 prime-target pairs. Primes were visually presented in silence, i.e., were presented as unlabeled, familiar images. Targets were presented auditorily, i.e., the picture prime was followed by an auditorily presented target word. A female, native speaker of German recorded all target words. The relationship between the labels for the prime image and the auditory target labels was manipulated to create four conditions: identity_-picture prime labels and L1 target words were identical in German (e. g., prime picture monkey "Affe" - target word Affe), within-language condition-L1 German labels for the picture primes and L1 target words were phonologically related in German (e. g., prime picture flag "Fahne"- target word Sahne "cream"), between-language condition-L2 English labels for the picture primes and L1 target words sounded similar (e. g., prime picture slide "Rutsche"target word Kleid "dress"), or unrelated-L1 and L2 labels for the picture prime and L1 target words were phonologically, orthographically, or semantically unrelated (e. g., prime picture knife "Messer"-target word Seil "rope"). The 120 prime-target pairs were distributed across the four conditions with 30 pairs per condition. Prime and target words across languages were matched on the frequency of the words as well as the number of syllables and phonemes in the words ( $p$ 's $>0.05$ ). Figure 1 contains example stimuli from each condition. A list of stimuli can be found in the Supplementary Material. 


\section{PROCEDURE}

\section{Main experiment}

Participants were seated in a dimly lit, quiet experimental room, facing a $92 \mathrm{~cm}$ wide and $50 \mathrm{~cm}$ high TV screen at a distance of $100 \mathrm{~cm}$ from the screen. All instructions given to participants, including the written instructions presented on an instruction sheet, were in German. Participants were presented with 120 trials distributed across the four conditions, with 30 trials per condition. Each trial began with a fixation cross displayed in the center of the screen for $1000 \mathrm{~ms}$. Following the offset of the fixation cross, participants were presented with the prime image centrally located on the screen. The prime image remained on screen for $500 \mathrm{~ms}$ (from 1000 to $1500 \mathrm{~ms}$ into the trial) followed by a blank, black screen. At $1550 \mathrm{~ms}$ into the trial, $50 \mathrm{~ms}$ after the offset of the prime picture, participants were presented with an auditory target word. At $3000 \mathrm{~ms}$ (i.e., $1450 \mathrm{~ms}$ after the onset of the target label), a second image was displayed that was either identical to the prime image or was different to the prime image. This image remained on-screen for $500 \mathrm{~ms}$ (from 3000 to $3500 \mathrm{~ms}$ into the trial) and was followed by a blank, black screen for a further $1000 \mathrm{~ms}$ (from 3500 to $4500 \mathrm{~ms}$ into the trial). Participants were instructed to indicate in this interval (from 3500 to $4500 \mathrm{~ms}$ into the trial) whether the second image matched the first image presented or not, by pressing one of two buttons in front of them. Participants were informed that the experiment investigated the mechanisms underlying their perception of the similarity between the two images presented. They were informed that they would hear spoken words during the experiment but that their task was to ignore these spoken words. This was done in order to avoid any overt attention to the relationship between the labels for the prime images and the target words.

\section{Production task}

Following the main experiment, participants also completed a production task, where they were asked to label a series of 60 images aloud in both German and English. Half of the participants labeled the images in German first, while the other half labeled in English first. Stimuli used in the production task were the prime images from the within- and between-language conditions. The answers provided by the participants were automatically recorded via a microphone, time-locked to the appearance of the image on-screen. Production data was analyzed offline to determine whether participants labeled the prime images from these conditions with the label we had chosen for each picture. If the label provided by a participant was different from the chosen label for an image, then the trial containing this image was removed from the main experiment. This was to ensure that the labels implicitly generated by individual participants were related to the target words in the two critical conditions. For example, in the between-language condition, the picture prime "beagle" could also be given the label "dog." However, the label "dog" does not sound similar to the target word "Igel" and therefore no longer fulfills the between-language manipulation. For the betweenlanguage condition, this removed $13 \%$ of trials ( 71 trials) and for the within-language condition $10.34 \%$ of trials $(60 \text { trials })^{2}$.

\footnotetext{
${ }^{2}$ An analysis on the data without removing trials that were produced correctly showed the same pattern of results as the analysis reported.
}

\section{EEG RECORDING AND ANALYSIS}

Electrophysiological data was recorded using the Biosemi Active Two Amplifier system at a sampling rate of $2048 \mathrm{~Hz}$ from $32 \mathrm{Ag} / \mathrm{AgCl}$ electrodes placed according to the $10-20$ convention. Electrode offsets were kept at less than $25 \mu \mathrm{V}$. Electroencephalogram was re-referenced offline to the averaged mastoid reference. EEG data was then filtered off-line using a $0.1 \mathrm{~Hz}$ high-pass forward filter and a $20 \mathrm{~Hz}$ low-pass, zero-phase shift filter.

Averaging and artifact rejection were carried out using the BESA software (Version 5.3). Blink and movement artifacts were automatically rejected using a $100 \mathrm{~Hz}$ amplitude cut-off across all electrodes. Epochs were defined from -200 to $1000 \mathrm{~ms}$ from the onset of the auditory target word. We then analyzed the data in $50 \mathrm{~ms}$ time windows (from 0 to $1000 \mathrm{~ms}$ ) to determine the onset and offset of significant differences between conditions. Based on this analysis, and the known onset of the N400 (Kutas and Hillyard, 1984), we focused our analyses on the time window between 300 and $400 \mathrm{~ms}$ (the N400 window; Desroches et al., 2009).

Average ERP waveforms were quantified by computing mean amplitudes per subject, electrode and condition in the selected time windows. ERP waveforms were baseline corrected by subtracting the mean amplitude for the baseline time window ( -200 to $0 \mathrm{~ms}$ ) from the selected time window. For purposes of data reduction, a selection of electrode locations was entered into data analysis, 16 electrodes divided into four regions and four lateral columns: frontal (F7, F3, F4, F8), fronto-central (FC5, FC1, FC2, FC6), central (T7, C3, C4, T8), and centroparietal (CP5, CP1, CP2, CP6). Our analysis was based on specific planned comparisons between related conditions (identical, within- and between-language conditions) and the unrelated condition instead of a general condition effect; we therefore do not report the omnibus ANOVA (see Abelson and Prentice, 1997). Factors included in the repeated measures ANOVA were region (frontal, fronto-central, central, and centro-parietal), electrode laterality (4), and condition (2; related, unrelated).

\section{RESULTS}

Figure 2 displays ERP waveforms aggregated across electrodes, separated by region (frontal, fronto-central, central, centroparietal) for each of the three condition comparisons (identity vs. unrelated/within-language vs. unrelated/between-language vs. unrelated). We first examined the difference in the mean N400 amplitude of the brain potentials following identity and unrelated prime-target pairs. Here, a significant main effect of condition revealed that mean N400 amplitude was reduced for identity prime-target pairs relative to unrelated prime-target pairs, $F_{(1,15)}=6.20, p=0.025, \eta_{p}^{2}=0.29$. No other interactions with condition were significant ( $p s>0.25$ ). Planned post-hoc analyses revealed that mean $\mathrm{N} 400$ amplitude for identity primetarget pairs was reduced relative to unrelated prime-target pairs across all regions, i.e., at frontal, $t_{(15)}=2.23, p=0.042, d=$ 0.25 , fronto-central, $t_{(15)}=2.95, p=0.01, d=0.37$, and central $t_{(15)}=2.46, p=0.026, d=0.43$, regions, and approached significance for the centro-parietal region, $t_{(15)}=1.92, p=0.074$, $d=0.48$. As expected, complete match between the label for the prime image and the target word in the identity condition resulted 


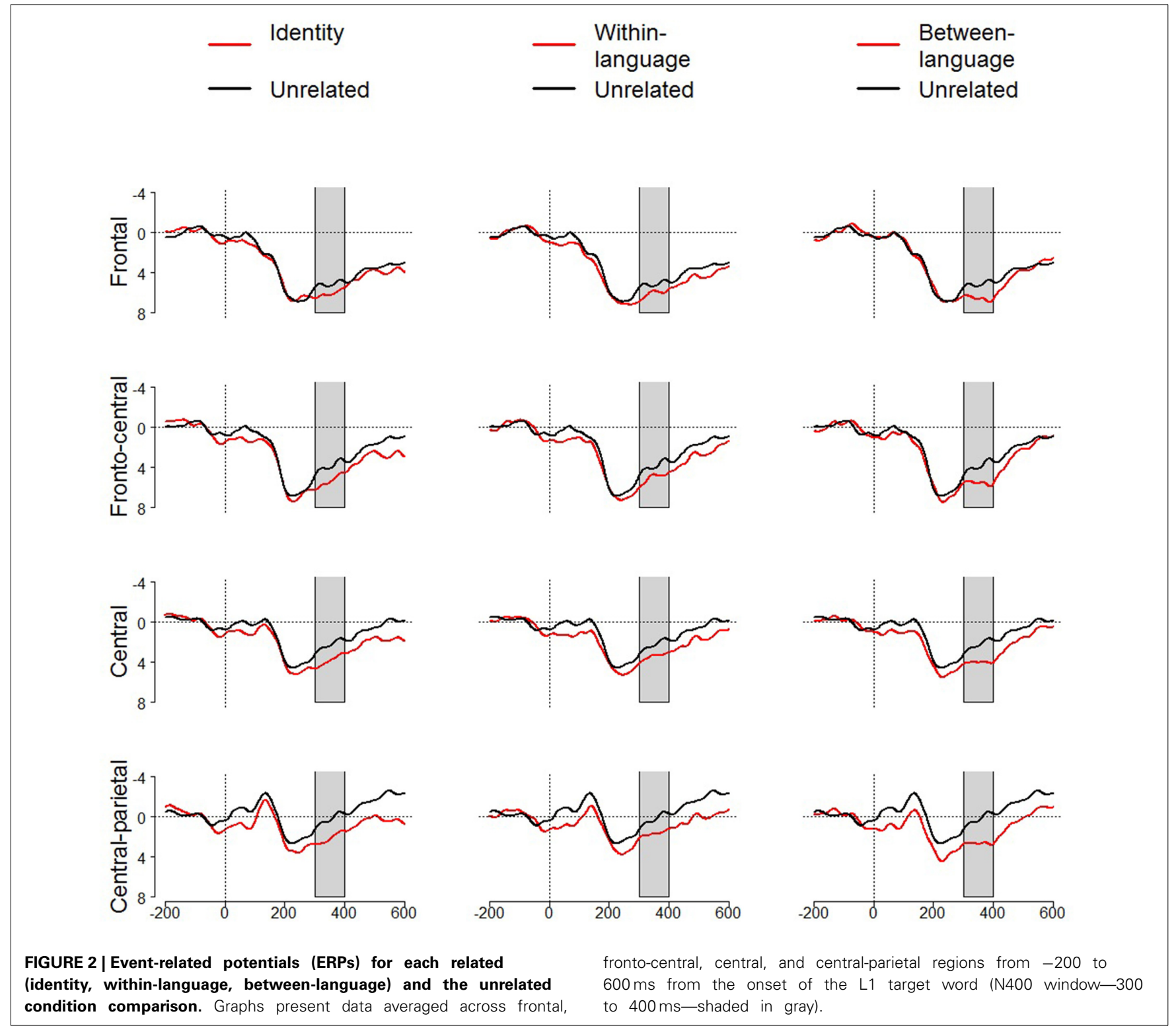

in easier processing of the target word, relative to when the prime label was unrelated to the target word.

Next, we examined the difference in mean N400 amplitude for within-language related prime-target pairs and unrelated primetarget pairs. A repeated-measures ANOVA revealed a significant main effect of condition, $F_{(1,15)}=4.54, p=0.05, \eta_{p}^{2}=0.23$, suggesting less negative N400 amplitude to L1 targets preceded by primes whose L1 labels were phonologically related to the L1 target words, relative to targets preceded by unrelated primes. No other interactions with condition were significant ( $p$ 's $>$ 0.56). Planned post-hoc analyses revealed that mean N400 amplitude for within-language prime-target pairs was reduced, relative to unrelated prime-target pairs, across all regions, significant at frontal, $t_{(15)}=2.16, p=0.047, d=0.23$, and fronto-central, $t_{(15)}=2.25, p=0.04, d=0.24$, regions and approached significance at the centro-parietal region, $t_{(15)}=1.83, p=0.088$, $d=0.32$, but not the central region $(p>0.12)$. In line with predictions, participants implicitly generated the label for the prime image in their L1, which speeded processing of the phonologically related L1 target word.

Finally, we examined the difference in mean N400 amplitude for between-language related prime-target pairs and unrelated prime-target pairs. An ANOVA comparing N400 amplitude across between-language related pairs and unrelated primetarget pairs revealed a near-significant main effect of condition, $F_{(1,15)}=4.25, p=0.057, \eta_{p}^{2}=0.22$. No other interactions with condition were significant $(p$ 's $>0.18)$. Planned post-hoc analyses revealed that mean N400 amplitude for between-language primetarget pairs was reduced, relative to unrelated prime-target pairs, across all regions, significant at central, $t_{(15)}=2.37, p=0.032$, $d=0.40$, and centro-parietal, $t_{(15)}=2.13, p=0.05, d=0.53$, regions, but not frontal or fronto-cental regions ( $p$ 's $>0.12$ ). The only way that the prime image in between-language related prime-target pairs could influence recognition of the target, 
would be if participants were to also implicitly generate the label for the prime image in their L2, and for this implicitly generated L2 label to speed processing of the phonologically related L1 target word.

To examine whether there was any differences in the magnitude of the priming effect across related conditions, further analyses compared ERPs to targets between the related conditions. ANOVAs comparing N400 amplitude for identity and within-language, identity and between-language, and within- and between-language prime-target pairs revealed no significant main effect of condition $(p>0.38)$ or interactions with condition ( $p s>0.15$ ), except for a significant interaction between condition $\mathrm{X}$ electrode laterality for the comparison between the identity and between-language conditions, $F_{(3,45)}=3.76, p=0.031, \eta_{p}^{2}=$ 0.20 . Paired-samples $t$-tests, however, revealed that there was no significant difference between the identity and between-language conditions at each of the lateral columns ( $p$ 's $>0.3$ ). The lack of a significant difference between the related conditions suggests a similar magnitude in the priming effect independent of a withinor between-language relationship between prime labels and target words.

In addition, we also measured the accuracy with which participants labeled the within and between-language related primes in German and English as well as the latency to name the images. In this task, conducted after the main experiment, participants were asked to overtly name the prime pictures from the within- and between-language conditions in German and English. Trials with no response were considered technical errors (German: 1.67\%; English: $3.13 \%$ ) and not included in the analysis. We considered responses correct if they accurately labeled the picture, regardless of the label we chose for the picture (e.g., cat or kitty for the prime picture kitten). Participants gave an incorrect response for $2.50 \%$ of trials in German and 2.70\% of trials in English. Trials containing images whose labels participants incorrectly labeled were excluded from the analysis. There was no difference in accuracy for participants when they labeled pictures in German (96.83\%) or English $(94.17 \% ; p>.3)$. In addition, there was no difference in reaction time for pictures named in German $(M=712.20 \mathrm{~ms}$; $S D=188.24 \mathrm{~ms})$ and English $(M=728.00 \mathrm{~ms} ; S D=185.05 \mathrm{~ms}$; $p>0.75)$. Taken together, participants showed no difference between German and English in the production task.

\section{DISCUSSION}

The current study asked whether bilingual adults implicitly generate the label for words in one or both of their languages, and whether implicitly generated L2 labels can prime L1 auditory words. These findings suggest that bilingual adults implicitly generate the label for visually fixated images in both of their languages, and that this implicitly generated label can, subsequently influence recognition of an auditorily presented, similar sounding L1 target word. These results significantly extend previous findings to strongly support suggestions that (a) implicit generation of the labels of visually fixated images in both languages of bilinguals immersed in their L1 environment and (b) L2 prime labels influencing recognition of L1 target words, despite the experiment being carried out in an L1 environment with only L1 stimuli being used in the experiment. This presents a robust test of the extent to which bilinguals implicitly generate picture labels in their L2 as well as their L1 in an experiment situation that discourages such activation.

\section{PICTURE LABELS ARE IMPLICITLY GENERATED IN L1 AND L2}

Prime-target pairs whose labels were either identical (identity; i.e., prime picture monkey "Affe" — target word Affe) or phonologically related within L1 (within-language; i.e., prime picture flag "Fahne"-target word Sahne "cream") elicited a reduction in N400 amplitude, suggesting that participants implicitly generated the label for prime pictures in their L1. That is, the implicitly generated L1 label subsequently primed the L1 target word as a result of the complete or phonological overlap between prime and target. This replicates previous studies with monolingual adults (Meyer et al., 2007; Desroches et al., 2009) and infants (Mani and Plunkett, 2010, 2011) that show that prime pictures presented in silence activate their labels and corresponding phonological information, priming subsequently presented identical or phonologically-related targets.

Critical to the current study's research questions, the reduction in N400 amplitude for L1 target words preceded by prime pictures whose label in L2 English was similar sounding to the L1 target word (between-language; i.e., prime picture slide "Rutsche"target word Kleid "dress"), suggests that bilingual participants also implicitly generated the prime picture label in their L2. This demonstrates that bilinguals implicitly generate the labels for objects in not one, but both of their languages. In a previous study, Wu and Thierry (2011) asked Chinese-English bilinguals to preform rhyme judgments on picture pairs, some of which were rhyme pairs in either Chinese or English. Priming effects were elicited for rhyme pairs in both languages when participants were tested in L2 English, but when participants were tested in L1 Chinese, only Chinese rhyme pairs elicited priming effects. The results of $\mathrm{Wu}$ and Thierry suggest that whether participants implicitly generated picture labels in one or both languages, therefore, depended on the language they were tested in. Wu and Thierry conclude that this asymmetry shows that spoken language planning (i.e., implicit label generation) in L1 proceeds without activating L2 word information, but that bilinguals are unable to prevent L1 interference during L2 speech planning.

In the current study, however, we find that when participants are tested in their L1, they implicitly generate the label not only in $\mathrm{L} 1$, as $\mathrm{Wu}$ and Thierry found, but also in L2. We suggest that the difference between the current study and that of Wu and Thierry is the result of the tasks which participants completed during the experiment. The bilingual participants tested by $\mathrm{Wu}$ and Thierry were instructed to make rhyme judgments for picture pairs. This task required participants to focus on the linguistic relationship between the prime and target pictures and narrow their focus to one language in order to successfully complete the task. When tested in L1, participants were better at narrowing their focus and preventing interference from L2, but this was not the case when participants were test in $\mathrm{L} 2$ and as a result $\mathrm{L} 1$ words were also activated. In the current study, bilingual participants judged whether the picture prime and a subsequently presented picture (after the target word) were the same or different. This task did not require participants to pay attention to the relationship between picture 
prime and target word. Unlike the participants tested by $\mathrm{Wu}$ and Thierry, the participants tested in the current study did not need to narrow their focus to one language in order to successfully complete their task. We suggest that this difference in task is the reason we find implicit label generation in both languages when participants were tested in L1, while Wu and Thierry did not. It is especially useful for future research that implicit label generation in bilinguals can be studied without using a task that calls attention to the relationship between prime and target.

We note that our results also contrast with previous work by Weber and Cutler (2004) who report that bilinguals do not activate their L2 in L1 processing, when immersed in an L1 dominant environment. In our study, similar to Weber and Cutler (2004), participants were immersed in their L1 and tested in their L1. In this situation, the only relevant language is L1. Yet, our results demonstrate that participants implicitly generated the label for prime pictures in both L1 and L2. One possible explanation is the potential difference in L1 and L2 use between the Dutch-English bilinguals tested by Weber and Cutler, and the German-English bilinguals tested in the current study. Previous studies have found that an L2 immersion context has a positive influence on L2 proficiency and performance compared to L2 classroom exposure, although this comes at the cost of L1 fluency (Linck et al., 2009; see also Baus et al., 2013). Faced with the task of L2 usage every day, bilinguals may inhibit their L1 (Green, 1998) in order to perform successfully in their L1 or this may simply be the result of reduced frequency of L1 use (Gollan et al., 2005, 2008). In the context of L1 immersion, L2 fluency may also experience a reduction, which would account for the findings of Weber and Cutler but not those of the current study. The difference, then, would lie in the usage of L2 English in the different L1 contexts of Dutch and English. Although comparisons of English proficiency and frequency of use across cultures are at the moment anecdotal at best, such considerations are crucial to the future study of bilingual language processing. Alternatively, it is possible that the use of a more sensitive paradigm to assess participants' access to L2 words, i.e., the cross-modal ERP priming paradigm, may have allowed us to tap into cross-language effects that could not be observed in Weber and Cutler. Indeed, a number of studies have shown that such subtle cross-language effects do not lead to noticeable differences in responding while triggering different patterns of neural activity (Kotz and Elston-Güttler, 2004; McLaughlin et al., 2004; Tokowicz and MacWhinney, 2005; Thierry and Wu, 2007; Wu and Thierry, 2010a; for a review see Mueller, 2005).

We suggest that, while future studies are needed to compare different language combinations and environments, the crossmodal ERP priming paradigm used in the current study is useful tool for measuring bilingual co-activation and allows us to tap into subtle effects of other language activation in bilingual language processing. This finding, taken together with other studies using different methods (Spivey and Marian, 1999; Wu and Thierry, 2011; Von Holzen and Mani, 2012) suggests that activation of both languages during processing in one language is a powerful phenomenon. While viewing pictures, bilinguals activate the corresponding label as well as its phonological information in both of their languages. Although such a result cannot be generalized outside of an experimental setting, it affords a glimpse into the complex processes that bilinguals undergo while interacting with their environment.

\section{CROSS-LANGUAGE PRIMING IN AN L1 EXPERIMENTAL SETTING}

The current study also provides evidence for cross-language priming in bilinguals such that implicitly generated L2 labels facilitate recognition of auditory L1 targets despite the experiment being conducted entirely in one language. Although relatively unexplored in auditory word processing (but see Phillips et al., 2006; Pratt et al., 2013), previous studies have also found similar L2-L1 priming effects for phonologically related prime-target pairs in visual word processing (Van Wijnendaele and Brysbaert, 2002; Duyck, 2005; Zhou et al., 2010). These studies, however, used both languages in their experiments, and priming effects may, therefore, result from the artificial bilingual environment created by using both languages in the experimental setting (Grosjean, 1997). In contrast, the current study was conducted in one language. The between-language priming effect, therefore, cannot be attributed to an artificial bilingual environmental setting and the current results present the first evidence for L2L1 priming in auditory word processing in an unbiased setting. This a useful tool for future studies to continue studying crosslanguage phono-lexical effects in bilinguals without presenting word stimuli auditorily or visually in both languages.

\section{IMPLICATIONS FOR MODELS OF LEXICAL ACCESS DURING SPEECH PRODUCTION}

The two main findings of the current study, namely that bilinguals implicitly generate the labels for pictures in both of their languages and cross-language phonological priming in participants immersed in their L1, provide an interesting addition to the debate on the kind of information that is activated during word production. It is generally accepted that speech production involves first activating the lexical node associated with the concept, followed by the corresponding phonological code (Levelt, 1989; Roelofs, 1992; Caramazza, 1997; Dell et al., 1997). Conflict abounds, however, with regard to whether the phonological information of non-selected lexical nodes is also activated. Discrete serial models of lexical access suggest that only the phonological information associated with the selected lexical node is activated (Levelt, 1989; Levelt et al., 1999). For example, when naming a picture of a table, semantically related lexical nodes are also activated (i.e., couch, chair). Ultimately, the lexical node for table is selected and the corresponding phonological information is activated, but not for the non-selected lexical nodes (i.e., couch, chair). Cascaded activation models of lexical access, in contrast, propose that the phonological information from both the selected and non-selected lexical nodes is activated (Dell, 1986; Caramazza, 1997; Dell et al., 1997).

The findings of the current study and Wu and Thierry (2011), provide useful information with regard to the processes underlying picture naming and, by extension, the extent to which conceptual and phonological levels of representations are recruited in speaking. Neither of these studies used prime-target pairs with semantic overlap (although Wu and Thierry did include a semantically related condition, prime-target pairs in the critical 
rhyming conditions were not additionally semantically related). Nevertheless, both studies demonstrate that participants activate phonological information associated with non-selected lexical nodes in implicit generation of the labels for visually fixated images. In other words, participants activated phonological information associated with both $\mathrm{L} 1$ and $\mathrm{L} 2$ labels for the images. If purely semantic information associated with non-selected lexical nodes were activated, as suggested by discrete serial models (Levelt, 1989; Levelt et al., 1999), we would have expected priming effects only in the identity condition. But, the priming effect for the between-language condition in the current study provides support for cascaded activation models of lexical access (Dell, 1986; Caramazza, 1997; Dell et al., 1997), by demonstrating that phonological information from both selected (L1) and nonselected (L2) lexical nodes was activated when our participant viewed the prime pictures. Our study, furthermore, goes beyond Wu and Thierry (2011) in showing that participants activated L2 labels for the prime images despite being immersed in their L1 and tested in their L1, thereby reducing the likelihood that L2 lexical nodes would need to be retrieved.

\section{CONCEPTUAL ACTIVATION DURING IMPLICIT LABEL GENERATION IN BILINGUALS}

The results of the current study have interesting implications for models of bilingual speech processing with regard to the activation of language representations from conceptual representations during picture viewing. The Revised Hierarchical Model is a model of bilingual word production that focuses on the connections between L1 and L2 words at the lexical and conceptual levels and how these connections develop as proficiency increases (Kroll and Stewart, 1994; Kroll and Dijkstra, 2002; Kroll et al., 2010). According to this model, connections exist between L1 and L2 translation equivalents at the lexical level. At the conceptual level, however, connections initially exist only between L1 words and their concepts. Access to conceptual representations during L2 processing must, therefore, be mediated through L1 translation equivalents. With greater L2 proficiency, access to conceptual representations during $\mathrm{L} 2$ processing may continue without mediation through L1 translation equivalents, with direct links between L2 words and their conceptual representations.

Models of speech production argue that upon viewing a picture, participants activate the conceptual representations associated with this image, leading to phonological activation of either one or many selected lexical nodes associated with the activated conceptual representations. Thus, evidence of the activation of the L2 label for the image might be taken to suggest that, in proficient bilinguals, conceptual representations activated are directly linked to L2 labels such that viewing the picture leads to activation of conceptual representations which in turn directly activate both L1 and L2 labels. Alternatively, it is possible that, even in proficient bilinguals, L2 words are only indirectly linked to conceptual representations such that the results of the current study are explained as follows: viewing the picture leads to activation of conceptual representations, which in turn activate the L1 label, leading to mediated activation of the L2 label from the L1 label. While our study cannot rule out this explanation entirely, we note that there were no differences in the time-course or strength of the effects across the within-language and between-language overlap conditions, suggesting that such mediated activation through L1 labels is unlikely. To this extent, the results of the current study support the suggestion of the RHM that, in proficient bilinguals, L2 words are directly linked to their concepts.

Alternatively, we note that the results could also be explained without relying on access to the conceptual level. For example, the visual features of a picture may directly activate its' label, such that, upon viewing a picture, the visual features of the picture activate the corresponding labels in both languages. Activation of word labels from picture viewing relies, in this case, not on the activation of conceptual representations but rather on the recognition of the visual features of an image. It will be interesting, therefore, for future studies to explore the extent to which conceptual representations are involved in the progression from image recognition to implicit naming/production. We also note that regardless of whether L2 labels were activated directly from conceptual representations or from the L1 labels, the results of the current study strongly suggest that bilinguals implicitly produce the labels for visually fixated images in both their languages, even when immersed in an L1 setting and when tested in their dominant language.

\section{CONCLUSION}

The current study presented evidence that bilinguals implicitly generate the label for pictures in both of their languages. Previous studies have shown mixed results, suggesting that an L1 language environment (visual world paradigm; Weber and Cutler, 2004) or experimental task that requires participants to focus on the linguistic relationship between prime and target (rhyming judgment task; Wu and Thierry, 2011) may prevent co-activation of L2 words. By using a cross-modal ERP priming paradigm, we demonstrated not only that bilinguals implicitly generate the label for pictures in both of their languages, but also that implicitly generated L2 labels can prime related L1 words. The results provide support for cascaded activation models of lexical access, showing that phonological information associated with non-selected lexical nodes is retrieved during (implicit) picture naming.

\section{ACKNOWLEDGMENTS}

This work was funded by the German Excellence Initiative Award to Georg-August-Universität Göttingen (Third funding line: Institutional Strategy). The authors would like to thank Nicole Altvater-Mackensen and Susan Bobb for their helpful comments on earlier version of this manuscript as well as all of the subjects who participated in the study.

\section{SUPPLEMENTARY MATERIAL}

The Supplementary Material for this article can be found online at: http://www.frontiersin.org/journal/10.3389/fpsyg. 2014.01415/abstract

\section{REFERENCES}

Abelson, R. P., and Prentice, D. A. (1997). Contrast tests of interaction hypotheses. Psychol. Methods 2, 315-328.

Altvater-Mackensen, N., and Mani, N. (2011). "Bilinguals activate words from both languages when listening to spoken sentences: evidence from an ERPstudy," in Proceedings of the 33rd Annual Meeting of the Cognitive Science Society, 
eds L. Carlson, C. Hoelscher, and T. F. Shipley (Austin, TX: Cognitive Science Society), 1382-1387.

Alvarez, R. P., Holcomb, P. J., and Grainger, J. (2003). Accessing word meaning in two languages: an event-related brain potential study of beginning bilinguals. Brain Lang. 87, 290-304. doi: 10.1016/S0093-934X(03)00108-1

Baus, C., Costa, A., and Carreiras, M. (2013). On the effects of second language immersion on first language production. Acta Psychol. 142, 402-409. doi: 10.1016/j.actpsy.2013.01.010

Blumenfeld, H. K., and Marian, V. (2005). "Covert bilingual language activation through cognate word processing: an eye-tracking study," in Proceedings of the XXVII Annual Conference of the Cognitive Science Society (Stresa), 286-291.

Blumenfeld, H. K., and Marian, V. (2007). Constraints on parallel activation in bilingual spoken language processing: examining proficiency and lexical status using eye-tracking. Lang. Cogn. Proces. 22, 633-660. doi: 10.1080/01690960601000746

Canseco-Gonzalez, E., Brehm, L., Brick, C. A., Brown-Schmidt, S., Fischer, K., and Wagner, K. (2010). Carpet or Cárcel: the effect of age of acquisition and language mode on bilingual lexical access. Lang. Cogn. Proces. 25, 669-705. doi $10.1080 / 01690960903474912$

Caramazza, A. (1997). How many levels of processing are there in lexical access? Cogn. Neuropsychol. 14, 177-208. doi: 10.1080/026432997381664

Colomé, À. (2001). Lexical activation in bilinguals' speech production: language-specific or language-independent? J. Mem. Lang. 45, 721-736. doi: 10.1006/jmla.2001.2793

Colomé, À., and Miozzo, M. (2010). Which words are activated during bilingual word production? J. Exp. Psychol. Learn. Mem. Cogn. 36, 96-109. doi: $10.1037 / \mathrm{a} 0017677$

Costa, A., Caramazza, A., and Sebastian-Gallés, N. (2000). The cognate facilitation effect: implications for models of lexical access. J. Exp. Psychol. Learn. Mem. Cogn. 26, 1283-1296. doi: 10.1037/0278-7393.26.5.1283

Costa, A., Strijkers, K., Martin, C. D., and Thierry, G. (2009). The time course of word retrieval revealed by event-related brain potentials during overt speech Proc. Natl. Acad. Sci. U.S.A. 106, 21442-21446. doi: 10.1073/pnas.0908921106

Cutler, A., Weber, A., and Otake, T. (2006). Asymmetric mapping from phonetic to lexical representations in second-language listening. J. Phon. 34, 269-284. doi: 10.1016/j.wocn.2005.06.002

Dahan, D., and Tanenhaus, M. K. (2004). Continuous mapping from sound to meaning in spoken-language comprehension: immediate effects of verbbased thematic constraints. J. Exp. Psychol. Lear. Mem. Cogn. 30, 498-513. doi: 10.1037/0278-7393.30.2.498

De Bruijn, E. R. A., Dijkstra, T., Chwilla, D. J., and Schriefers, H. J. (2001). Language context effects on interlingual homograph recognition: evidence from eventrelated potentials and response times in semantic priming. Biling. Lan. Cogn. 4 155-168. doi: 10.1017/S1366728901000256

Dell, G. S. (1986). A spreading-activation theory of retrieval in sentence production. Psychol. Rev. 93, 283-321.

Dell, G. S., Schwartz, M. F., Martin, N., Saffran, E. M., and Gagnon, D. A. (1997) Lexical access in aphasic and nonaphasic speakers. Psychol. Rev. 104, 801-838.

Desroches, A. S., Newman, R. L., and Joanisse, M. F. (2009). Investigating the time course of spoken word recognition: electrophysiological evidence for the influences of phonological similarity. J. Cogn. Neurosci. 21, 1893-1906. doi 10.1162/jocn.2008.21142

Dijkstra, T., Grainger, J., and van Heuven, W. J. B. (1999). Recognition of cognates and interlingual homographs: the neglected role of phonology. J. Mem. Lang. 41, 496-518. doi: 10.1006/jmla.1999.2654

Duyck, W. (2005). Translation and associative priming with cross-lingual pseudohomophones: evidence for nonselective phonological activation in bilinguals. J. Exp. Psychol. Learn. Mem. Cogn. 31, 1340-1359. doi: 10.1037/02787393.31.6.1340

FitzPatrick, I., and Indefrey, P. (2010). Lexical competition in nonnative speech comprehension. J. Cogn. Neurosci. 22, 1165-1178. doi: 10.1162/jocn.2009.21301

FitzPatrick, I., and Indefrey, P. (2014). Head start for target language in bilingual listening. Brain Res. 1542, 111-130. doi: 10.1016/j.brainres.2013.10.014

Gollan, T. H., Montoya, R., Cera, C., and Sandoval, T. (2008). More use almost always means a smaller frequency effect: aging, bilingualism, and the weaker links hypothesis. J. Mem. Lang. 58, 787-814. doi: 10.1016/j.jml.2007.07.001

Gollan, T. H., Montoya, R. I., Fennema-Notestine, C., and Morris, S. K. (2005). Bilingualism affects picture naming but not picture classification. Mem. Cogn. 33, 1220-1234. doi: 10.3758/BF03193224
Green, D. (1998). Mental control of the bilingual lexico-semantic system. Biling. Lang. Cogn. 1, 67-81. doi: 10.1017/S1366728998000133

Grosjean, F. (1997). "Processing mixed language: issues, findings and models," in Tutorials in Bilingualism: Psycholinguistic Perspectives, eds A. M. B. de Groot and J. F. Kroll (Mahwah, NJ: LEA), 225-254.

Hermans, D., Bongaerts, T., De Bot, K., and Schreuder, R. (1998). Producing words in a foreign language: can speakers prevent interference from their first language? Biling. Lang. Cogn. 1, 213-229. doi: 10.1017/S1366728998000364

Hoshino, N., and Kroll, J. F. (2008). Cognate effects in picture naming: does crosslanguage activation survive a change of script? Cognition 106, 501-511. doi: 10.1016/j.cognition.2007.02.001

Hoshino, N., and Thierry, G. (2011). Language selection in bilingual word production: electrophysiological evidence for cross-language competition. Brain Res. 1371, 100-109. doi: 10.1016/j.brainres.2010.11.053

Jescheniak, J. D., Schriefers, H., Garrett, M. F., and Friederici, A. D. (2002). Exploring the activation of semantic and phonological codes during speech planning with event-related brain potentials. J. Cogn. Neurosci. 14, 951-964. doi: $10.1162 / 089892902760191162$

Ju, M., and Luce, P. A. (2004). Falling on sensitive ears: constraints on bilingual lexical activation. Psychol. Sci. 15, 314-318. doi: 10.1111/j.0956-7976.2004.00675.x

Kaushanskaya, M., and Marian, V. (2007). Bilingual language processing and interference in bilinguals: evidence from eye tracking and picture naming. Lang. Learn. 57, 119-163. doi: 10.1111/j.1467-9922.2007.00401.x

Kotz, S. A., and Elston-Güttler, K. (2004). The role of proficiency on processing categorical and associative information in the $\mathrm{L} 2$ as revealed by reaction times and event-related brain potentials. J. Neurolinguist. 17, 215-235. doi: 10.1016/S0911-6044(03)00058-7

Kroll, J. F., and Dijkstra, T. (2002). “The bilingual lexicon," in Handbook of Applied Linguistics, ed R. Kaplan (Oxford: Oxford University Press), 301-321.

Kroll, J. F., and Stewart, E. (1994). Category interference in translation and picture naming: evidence for asymmetric connections between bilingual memory representations. J. Mem. Lang. 33, 149-174. doi: 10.1006/jmla.1994.1008

Kroll, J. F., van Hell, J. G., Tokowicz, N., and Green, D. W. (2010). The revised hierarchical model: a critical review and assessment. Biling. Lang. Cogn. 13, 373-381. doi: $10.1017 /$ S136672891000009X

Kutas, M., and Hillyard, S. A. (1984). Brain potentials during reading reflect word expectancy and semantic association. Nature 307, 161-163. doi: $10.1038 / 307161 \mathrm{a} 0$

Lagrou, E., Hartsuiker, R. J., and Duyck, W. (2011). Knowledge of a second language influences auditory word recognition in the native language. J. Exp. Psychol. Learn. Mem. Cogn. 37, 952-965. doi: 10.1037/a0023217

Levelt, W. J. M. (1989). Speaking: From Intention to Articulation. Cambridge, MA MIT Press.

Levelt, W. J. M., Roelofs, A., and Meyer, A. S. (1999). A theory of lexical access in speech production. Behav. Brain Sci. 22, 1-75.

Linck, J., Kroll, J. F., and Sunderman, G. (2009). Losing access to the native language while immersed in a second language: evidence for the role of inhibition in second-language learning. Psychol. Sci. 20, 1507-1515. doi: 10.1111/j.14679280.2009.02480.x

Mani, N., Durrant, S., and Floccia, C. (2012a). Activation of phonological and semantic codes in toddlers. J. Mem. Lang. 66, 612-622. doi: 10.1016/j.jml.2012.03.003

Mani, N., Mills, D. L., and Plunkett, K. (2012b). Vowels in early words: an event-related potential study. Dev. Sci. 15, 2-11. doi: 10.1111/j.14677687.2011.01092.x

Mani, N., and Plunkett, K. (2010). In the infant's mind's ear: evidence for implicit naming in 18-month-olds. Psychol. Sci. 21, 908-913. doi: $10.1177 / 0956797610373371$

Mani, N., and Plunkett, K. (2011). Phonological priming and cohort effects in toddlers. Cognition 121, 196-206. doi: 10.1016/j.cognition.2011.06.013

Marian, V., Blumenfeld, H. K., and Boukrina, O. V. (2008). Sensitivity to phonological similarity within and across languages. J. Psycholinguist. Res. 37, 141-170. doi: 10.1007/s10936-007-9064-9

Marian, V., and Spivey, M. (2003a). Bilingual and monolingual processing of competing lexical items. Appl. Psycholinguist. 24, 173-193. doi: $10.1017 /$ S0142716403000092

Marian, V., and Spivey, M. (2003b). Competing activation in bilingual language processing: within- and between-language competition. Biling. Lang. Cogn. 6, 97-115. doi: 10.1017/S1366728903001068 
Martin, C. D., Dering, B., Thomas, E. M., and Thierry, G. (2009). Brain potentials reveal semantic priming in both the "active" and the "nonattended" language of early bilinguals. Neuroimage 47, 326-333. doi: 10.1016/j.neuroimage.2009.04.025

McLaughlin, J., Osterhout, L., and Kim, A. (2004). Neural correlates of secondlanguage word learning: minimal instruction produces rapid change. Nat. Neurosci. 7, 703-704. doi: 10.1038/nn1264

Meyer, A. S., Belke, E., Telling, A. L., and Humphreys, G. W. (2007). Early activation of object names in visual search. Psychon. Bull. Rev. 14, 710-716. doi: 10.3758/BF03196826

Meyer, A. S., and Damian, M. F. (2007). Activation of distractor names in the picture-picture interference paradigm. Mem. Cogn. 35, 494-503. doi: 10.3758/BF03193289

Moreno, E. M., Rodriguiez-Fornells, A., and Laine, M. (2008). Event-related potentials (ERPs) in the study of bilingual language processing. J. Neurolinguist. 21, 477-508. doi: 10.1016/j.jneuroling.2008.01.003

Mueller, J. L. (2005). Electrophysiological correlates of second language processing. Sec. Lang. Res. 21, 152-174. doi: 10.1191/0267658305sr256oa

Palmer, S. D., van Hooff, J. C., and Havelka, J. (2010). Language representation and processing in fluent bilinguals: electrophysiological evidence for asymmetric mapping in bilingual memory. Neuropsychologia 48, 1426-1437. doi: 10.1016/j.neuropsychologia.2010.01.010

Phillips, N. A., Klein, D., Mercier, J., and de Boysson, C. (2006). ERP measures of auditory word repetition and translation priming in bilinguals. Brain Res. 1125, 116-131. doi: 10.1016/j.brainres.2006.10.002

Phillips, N. A., Segalowitz, N., O’Brien, I., and Yamasaki, N. (2004). Semantic priming in a first and second language: evidence from reaction time variability and event-related brain potentials. J. Neurolinguist. 17, 237-262. doi: 10.1016/S0911-6044(03)00055-1

Poarch, G. J., and van Hell, J. G. (2012). Cross-language activation in children's speech production: evidence from second language learners, bilinguals, and trilinguals. J. Exp. Child Psychol. 111, 419-438. doi: 10.1016/j.jecp.2011.09.008

Pratt, H., Abbasi, D. A.-A., Bleich, N., Mittelman, N., and Starr, A. (2013). Spatiotemporal distribution of cortical processing of first and second languages in bilinguals. II. Effects of phonologic and semantic priming. Hum. Brain Mapp. 34, 2882-2898. doi: 10.1002/hbm.22109

Roelofs, A. (1992). A spreading-activation theory of lemma retrieval in speaking. Cognition 42, 107-142. doi: 10.1016/0010-0277(92)90041-F

Rueschemeyer, S.-A., Nojack, A., and Limbach, M. (2008). A mouse with a roof? Effects of phonological neighbors on processing of words in sentences in a nonnative language. Brain Lang. 104, 132-144. doi: 10.1016/j.bandl.2007.01.004

Shook, A., and Marian, V. (2012). The bilingual language interaction network for comprehension of speech. Biling. Lang. Cogn. 16, 304-324. doi: $10.1017 /$ S1366728912000466

Sinai, A., and Pratt, H. (2002). Electrophysiological evidence for priming in response to words and pseudowords in first and second language. Brain Lang. 80, 240-252. doi: 10.1006/brln.2001.2597

Sorensen, D. W., and Bailey, K. G. D. (2007). The world is too much: effects of array size on the link between language comprehension and eye movements. Visual Cogn. 14, 112-115. doi: 10.1080/13506280600975486

Spalek, K., Hoshino, N., Wu, Y. J., Damian, M., and Thierry, G. (2014). Speaking two languages at once: unconscious native word form access in second language production. Cognition 133, 226-231. doi: 10.1016/j.cognition.2014.06.016

Spivey, M. J., and Marian, V. (1999). Cross talk between native and second languages: partial activation of an irrelevant lexicon. Psychol. Sci. 10, 281-284. doi: $10.1111 / 1467-9280.00151$
Strijkers, K., Costa, A., and Thierry, G. (2010). Tracking lexical access in speech production: electrophysiological correlates of word frequency and cognate effects. Cereb. Cortex 20, 912-928. doi: 10.1093/cercor/bhp153

Thierry, G., and Wu, Y. J. (2007). Brain potentials reveal unconscious translation during foreign-language comprehension. Proc. Natl. Acad. Sci. U.S.A. 104 12530-12535. doi: 10.1073/pnas.0609927104

Tokowicz, N., and MacWhinney, B. (2005). Imiplicit and explicit measures of sensitivity to violations in second language grammar: an eventrelated potential investigation. Stud. Sec. Lang. Acquis. 27, 173-204. doi: $10.1017 /$ S0272263105050102

Van Wijnendaele, I., and Brysbaert, M. (2002). Visual word recognition in bilinguals: phonological priming from the second to the first language. J. Exp. Psychol. Hum. Percep. Perform. 28, 616-627. doi: 10.1037//0096-1523.28. 3.616

Von Holzen, K., and Mani, N. (2012). Language nonselective lexical access in bilingual toddlers. J. Exp. Child Psychol. 113, 569-586. doi: 10.1016/j.jecp.2012.08.001

Weber, A., and Broersma, M. (2012). "Spoken word recognition in second language acquisition," in The Encyclopedia of Applied Linguistics, ed C. A. Chapelle (Bognor Regis: Wiley-Blackwell), 5356-5361. doi: 10.1002/9781405198431.wbeal1104

Weber, A., and Cutler, A. (2004). Lexical competition in non-native spoken-word recognition. J. Mem. Lang. 50, 1-25. doi: 10.1016/S0749-596X(03)00105-0

Weber, A., and Paris, G. (2004). "The origin of the linguistic gender effect in spoken-word recognition: evidence from non-native listening," in Proceedings of the 26th Annual Meeting of the Cognitive Science Society, eds K. Forbus, D. Gentner, and T. Tegier (Mahwah, NJ: Erlbaum), 1446-1451.

$\mathrm{Wu}$, Y. J., and Thierry, G. (2010a). Chinese-english bilinguals reading english hear Chinese. J. Neurosci. 30, 7646-7651. doi: 10.1523/JNEUROSCI.160210.2010

Wu, Y. J., and Thierry, G. (2010b). Investigating bilingual processing: the neglected role of language processing contexts. Front. Psychol. 1:178. doi: 10.3389/fpsyg.2010.00178

Wu, Y. J., and Thierry, G. (2011). Event-related brain potential investigation of preparation for speech production in late bilinguals. Front. Psychol. 2:114. doi: 10.3389/fpsyg.2011.00114

Zhou, H., Chen, B., Yang, M., and Dunlap, S. (2010). Language nonselective access to phonological representations: evidence from Chinese-English bilinguals. Q. J. Exp. Psychol. 63, 2051-2066. doi: 10.1080/17470211003718705

Conflict of Interest Statement: The authors declare that the research was conducted in the absence of any commercial or financial relationships that could be construed as a potential conflict of interest.

Received: 10 June 2014; accepted: 19 November 2014; published online: 09 December 2014.

Citation: Von Holzen K and Mani N (2014) Bilinguals implicitly name objects in both their languages: an ERP study. Front. Psychol. 5:1415. doi: 10.3389/fpsyg.2014.01415 This article was submitted to Language Sciences, a section of the journal Frontiers in Psychology.

Copyright (c) 2014 Von Holzen and Mani. This is an open-access article distributed under the terms of the Creative Commons Attribution License (CC BY). The use, distribution or reproduction in other forums is permitted, provided the original author(s) or licensor are credited and that the original publication in this journal is cited, in accordance with accepted academic practice. No use, distribution or reproduction is permitted which does not comply with these terms. 